\title{
A Survey on RCF Based Data Dissemination Techniques for VANETs
}

\author{
E.Suneetha ${ }^{1}$ and S.Bharathi Priya ${ }^{2}$ \\ ${ }^{1}$ CVR College of Engineering, Department of CSE, Ibrahimpatan, R.R.District, A.P., India \\ Email: suneethaeluri9@gmail.com \\ ${ }^{2}$ CVR College of Engineering, Department of CSE, Ibrahimpatan, R.R.District, A.P., India \\ Email: bharathipriya.cs@gmail.com
}

\begin{abstract}
Vehicular Ad hoc Networks (VANETs) are the commercial version of Mobile Ad hoc Networks (MANETs) having very high mobility in which every vehicle acting as a host as well as router and forwards the packets to other vehicles. VANET forms decentralized networks, which enable plethora of important applications and services ranging from life safety applications to infotainment applications. High mobility in vehicular networks causes rapid topology changes and frequent disconnections. Due to this, data dissemination is a challenging task for researchers. Most of the data dissemination technique follows the iterative approach of Relay, Carry and Forward(RCF) in which road side units periodically pours the data packets to the moving vehicles, it buffers the data packet until a new vehicle enters into its vicinity and forwards the packet. This procedure establishes multi-hop communication to reach the destination. All these techniques have to consider about the delay and delivery ratio constraints with available bandwidth. The Performance of these techniques was highly affected by traffic density and data set size. In this paper, various data dissemination techniques and their performances, issues were analyzed thoroughly.
\end{abstract}

Index Terms-Vehicular Ad hoc networks, data dissemination, Road side units, Packet delivery ratio, Traffic density, Data set size.

\section{INTRODUCTION}

Intelligent Transportation Systems (ITS) have been deployed in the U.S., Europe and Asia. Existing ITS deployments are "Infrastructure heavy" in that they rely on roadside sensors, cameras, networks, etc. While such systems provide substantial benefit, deployment is very costly. In order to support ITS, vehicles are equipped with computing and communication capabilities, where every vehicle node acts as a host as well as router and forwarding the messages to other mobile nodes. This forms Vehicular Ad hoc Networks (VANETs) [5], the high mobility of vehicles causes rapid topology changes. Hence MANETs [5] protocols are not suitable for vehicle communication.
VANETs can be envisioned as a "Self-Organized networks" where communication is performed between Vehicle-to-Vehicle(V2V) and Vehicle-toRoad side unit (V2R). The roadside unit (RSU) is typically a data center with specialized wire-less interface, it maintains list of messages that has to be propagated to the vehicles on the road. At every intersection data packets are transferred to the RSU and the RSU delivers the data packet to the vehicles that can transfer this packet to the destination with minimum delay.

Vehicular Networks provides plethora of applications and services ranging from the life safety application to infotainment applications. For Example, Vehicular networks are considered as the perfect way to bring more comfort to the passengers and more safety to human life, by providing emergency warnings like accidents, speed limit, traffic condition, road conditions and any obstacles on the road etc. These are considered as critical life safety applications in which delay is not tolerable(Non-delay tolerant applications) [4].Emergency messages are short, that have delay ,delivery ratio constraints and do not really care about bandwidth. Whereas the infotainment applications like commercial advertisements, informing about stock prices, enquiring about the nearby parking stations, gas filling stations, restaurants etc are delay-tolerant. These services have constraints related to bandwidth [1].

Through these applications, we can see that VANET is very useful for disseminating data from an information source (data center) to many vehicles on the road. Recently researchers have begun to work on various data dissemination techniques to address the issues in the VANETs. Good data dissemination algorithm must address the characteristics of the vehicular network in which it will operate. Let us therefore consider the characteristics of $\mathrm{V} 2 \mathrm{~V}$ networks. Assume instrumented vehicles are equipped with on-board computing and wireless communication devices, a GPS device enabling the vehicle to track its 
geographical-temporal trajectory, a pre-stored digital map, and other sensors reporting crashes, engine statistics, etc. Due to the gradual nature of market penetration, only a fraction of the vehicles on the road will be instrumented Specifically, the term "penetration ratio" is defined as the fraction of vehicles on the road that are instrumented. Only instrumented vehicles participate in the V2V system. In the remaining sections of this paper, the term "vehicles" refers to instrumented vehicles only.

Vehicles exchange information with other vehicles within their short radio range and ad hoc wireless networks are used to propagate information. A V2V network is a special type of ad hoc network. Some unique characteristics [16] that differentiate it from other types of ad hoc networks include: (1) predictable, high mobility that can be exploited for system optimization (2)dynamic, rapidly changing topology (due to high mobility) (3)constrained, largely one-dimensional movement due to static roadway geometry (4) potentially large-scale (5) partitioned. The probability of endto-end connectivity decreases with distance is implicitly assumed for ad hoc networks (6) vehicles are not completely reliable (7) no significant power constraints, unlike sensor and other types of mobile networks where limited battery life is a major concern. These properties make V2V networks different and significantly affect their design.

Above mentioned unique characteristics of the VANETs brings out new research challenges to the research community. First, due to fast vehicle movement, the link topology changes rapidly [16]. As a result, many well-studied structures for efficient data dissemination, such as trees, clustering, and grids, are extremely hard to set up and maintain. Second, the conventional broadcast mechanism for data dissemination may lead to broadcast storm [13] because the network node density is usually quite high in an urban area and extremely dense during rush hours or traffic jams. Third, the vehicle mobility is partially predictable since it is limited by the traffic pattern and the road layout [5]. Data dissemination techniques should address these unique characteristics of the VANET.

In vehicular ad hoc network the delivery is not only single hop but multi hop delivery of data could be done and even the vehicle which is miles away from the destination can also query there request like - traffic condition in the city can be obtained by the vehicles when they are out of city[7]. In these situation vehicle can forward their request to the other vehicles and can receive the response in sathe seconds or in fraction of minutes. Many data dissemination protocols [1] have been proposed to disseminate information about obstacles information, traffic conditions and mishap on the roads.

In this paper we are presenting some available techniques which are based on pull and push based mechanism. The rest of the paper is organized as follows: Section-II describes several data dissemination techniques in the vehicular ad hoc networks and finally Section-III concludes this paper and explains the future.

\section{DATA DisSEMINATION TECHNIQUES}

\section{A. Introduction}

Data Dissemination is the process of broadcasting the data periodically to the vehicles on the road. Data Dissemination in fixed infrastructure can utilize well-established routing protocol for wired networks. But Data Dissemination techniques for vehicular networks remain as a challenging task to meet the design objectives. Some of the design objective includes low end to end delay, high reliability, low memory occupancy, high packet delivery ratio and maximum dissemination capacity with available bandwidth.

The network traffic density [3] in VANETs is expressed in terms of number of vehicles on the road. Some of the design objectives are highly affected by the high and low traffic density. Normally, in the urban areas where people population is more or in peak hours, the number of vehicles on road is preferable more. During dense traffic conditions, always a vehicle finds a forwarder to deliver the data packet to the nearby RSU or to the intended node to reach the destination[2]. Low traffic and intermediate traffic conditions can be seen in rural areas, during nonrush hours, in high way scenarios or in rural areas, in these circumstances the number of vehicles on the road is likely to be less. Due to this reason there is always frequent disconnections between the vehicles, hence the data cannot be propagated/disseminated to the intended node/RSU. This situation arises in sparsely connected networks [3].On the other hand, the high mobility of vehicular networks introduces the opportunities for mobile vehicles to connect with each other intermittently during moving. To deal with disconnection in sparsely connected network [3], the idea of relay, carry and forward [13] is adopted, where the vehicles carry the packet when appropriate route do not exist to forward the packet to the new receiver that moves into vicinity. Through Relay, Carry and Forward, the message can be delivered to the destination without end to 
end connection by establishing multi hop communication.

Semantics of data dissemination services [16] and their suitability for ITS applications are mentioned below. Four services that have immediate application are unicast, multicast, any cast and scan. Unicast with precise location means a message should be delivered to node $i$ in location $l$ before time $t$. Unicast with approximate location means sending a message to node $i$ before time $t_{l}$ while that node was last known to be at location $l$ with mobility $m$ at time $t_{2}$. Multicast means disseminating a message to all receivers in region $r$ before time $t$. Anycast means disseminating a message to one among a set of possible destinations (e.g., send to any police car) in region $r$ before time $t$. Scan is to have a message traverse region $r$ once before time $t$. In these services, location $l$ and region $r$ are used to direct the message to a geographical area. Time $t$ is determined by the nature of the message, e.g., when the information becomes obsolete, and serves to avoid the infinite looping of messages in the system. Other services can also be designed as variations or combinations of the above services.

To illustrate an application using these services, consider a vehicle (or a traffic signal controller) wishing to obtain information concerning some remote region. The vehicle/controller needing the information first queries its own proximity (multicast) to determine if a near-by vehicle happens to have this information. Any vehicle having such information can respond (unicast with approximate/precise location). If no one replies within a certain amount of time, the vehicle/controller sends a query to any vehicle in the remote region (any-cast). Receivers in the remote region with this information can respond. The response can be disseminated as unicast with approximate/precise location, or multicast if caching is desired. This scenario describes a pull approach. A push approach could also be used, e.g., vehicles encountering a crash or traffic congestion may send this information to a region using multicast.

Another application is mobile Internet access. Fixed location Internet gateways may be placed along roads. A vehicle wishing to access the Internet first propagates a query through a region for gateways (scan). Gateways receiving the query can respond to the requesting vehicle (unicast with approximate location). The requesting vehicle picks one responder and begins to interact with it. The communication from the vehicle to the gateway is unicast with exact location while the reverse direction is unicast with approximate location.

\section{B. Data Dissemination Schemes}

Data dissemination is a challenging task because with the available bandwidth, maximum data has to be disseminated over vehicular network. Some of the data dissemination techniques are based on push-based and pull-based mechanisms [6]. In push based data dissemination scheme data is managed by data center which collects the data from outside world and maintains the list of messages that is to be disseminated over network. In this scheme, data dissemination follows simple flooding algorithm [12] where data center periodically broadcast the data to the vehicle which enters into transmission range. Data center includes the header information in to the data packet. Format of the header is given below:

\begin{tabular}{|l|l|l|l|l|}
\hline $\begin{array}{l}\text { Source } \\
\text { id }\end{array}$ & $\begin{array}{l}\text { Source } \\
\text { location }\end{array}$ & $\begin{array}{l}\text { Forwarding } \\
\text { direction }\end{array}$ & $\begin{array}{l}\text { Packet } \\
\text { Generation }\end{array}$ & $\begin{array}{l}\text { Packet } \\
\text { Expiration } \\
\text { time }\end{array}$ \\
\hline
\end{tabular}

Fig. 1 Header format

The pull based dissemination scheme is mainly used by vehicles to query the data for the specific response from data center or from other vehicles. Pull based scheme is used by some specific users. In this scheme the data is managed by the data center and the vehicles which are moving on the road. When the vehicle needs any data query then firstly these vehicles sends beacon message to find the list of neighbor vehicles. These vehicles are already equipped with digital maps, having street level maps and traffic details like traffic density and vehicle speed on roads at different times [1]. The carry and forward mechanism is used to deliver the data in this approach. In this mechanism data packets are carried by the vehicles and when they found another vehicle[s] moving in the direction of destination in its range, it forwards that packet to this vehicle. This mechanism takes tolerable delay to transfer data to the destination.

In this approach data packets are mostly transferred using wireless channels but if the packet has to be transferred through the roads then those roads will be chosen for data transfer through which highly mobile vehicles are moving. Since the vehicular ad hoc network are unpredictable in nature, so optimal path for success-ful routing cannot be computed before sending the packet. So the dynamic path selection [3] is done throughout 
the packet forwarding process. Since, pull based mechanism is generally used for making queries and receiving the response. So this whole process is typically divided into two sub processes. a) Requesting data from moving vehicle to fixed location. b) Receiving response from fixed location to moving Vehicle.

Several Data dissemination protocols were proposed by researchers and they are generally classified in to two classes:1) Protocols for safety/emergency applications(e.g Warning messages about road accidents, obstacle on the road) that have delay and delivery ratio constraints 2) Protocols for infotainment applications (e.g. .advertisement applications) that have constraints related to bandwidth.

\section{Dissemination for safety applications}

Many dissemination protocols have been proposed to perform safety messaging to avoid mishaps. These protocols need to provide low endto end delay and high delivery ratio constraints [4]. Some of the protocols are specified below:

In [14] $\mathrm{Xu}$ et al presented Vehicle-to-Vehicle safety messaging in DSRC; this paper explores the feasibility of sending safety messages from vehicle to vehicle in short radio range. Safety messages are time sensitive and these messages should be received reliably with small delays. They have defined the Probability of Reception failure (PRF) as the probability a targeted receiver fails to receive a safety message within given time delay and collision free. To accomplish the above criteria, MAC protocols are used. But this approach needs a fine tuning mechanism to work based on the traffic densities[15].

Another interesting work was proposed a scheme, called Directional Propagation Protocol (DPP) [11] based on clustering algorithm to regroup the vehicles into clusters. In each group a header and trailer is elected as an in-charge for message propagation.DPP consists of 3 modules; a Custody Transfer protocol, an Inter Clustering protocol and Intra-Clustering Routing protocol. It uses store and forward module to handle the network disconnections. Unfortunately, this paper fails to address the procedure for header and trailer election.

In Spatio-Temporal Emergency Information Diss-emination (STEID) [9], the main goal of the work is to quickly disseminate traffic alerts to every vehicle that passes through an emergency zone during the life time of the emergency. To achieve this goal, they proposed hybrid network architecture consisting of WiFi clusters connected through proxy servers and cellular links. As this work is based on cluster formation and maintenance, there is considerable overhead in forming and maintaining cluster for short period of time.

In [8], focuses on the "Enhancement of Multihop Vehicular Broadcast (MHVB)".The enhancement procedure is carried out in two steps: by changing the shape of the backfire region in the algorithm and by introducing a new dynamic scheduling algorithm which prioritizes the packet transmission based on "processing" of the received packets from other vehicles. This work also includes traffic congestion detection algorithm by counting the number of vehicles surrounding a concerned node and detects the congestion. If it is the case, it expands the interval of transmitting its own information, therefore saving bandwidth and reducing collisions.

\section{D.Dissemination for infotainment applications}

The infotainment services (such as delivering announcing advertisements about sale promotions, getting information on the available parking places, and carpooling possibilities, etc.) interest mostly the network operators and service providers. The dissemination protocols used in such class of services have no constraints in terms of delay and delivery ratio (a good delay or delivery ratio is appreciated but not mandatory)[10]. However, they have constraints related to the bandwidth use. In the following section, some of the protocols are discussed with their performance issues.

In [12] Xu et al proposed an Opportunistic dissemination scheme in which data center periodically broadcast the data which will be received and stored by passing vehicle in the range of the data center. Whenever two vehicles move into the transmission range of each other, they exchange the data. This approach is followed for the user queries on the local database with predefined spatial and temporal boundaries. When the vehicle density is very high, every vehicle exchange their information with other vehicles in its range resulting in MAC layer collisions. This significantly reduces the data delivery ratio.

To mitigate excessive transmissions and congestions, KormaZ et al proposed [13] a link layer based protocol refereed as Urban Multihop Broadcast (UMB) protocol for inter-vehicle communication system. UMB is designed to address three problems: 1) Broadcast storm 2) Hidden node 3) Reliability. This protocol does not simply broadcast the data packet to all the neighboring nodes, instead it selects the farthest node in its broadcast direction's transmission range and assign the duty of forwarding the data and acknowledgement further. Repeaters are installed in 
the intersections to disseminate the packet in all directions. Basically this protocol is a segment based where the road portion is segmented into sub segments to select the forwarder. As a result, this protocol can adapt itself to light or heavy traffic condition. In order to decrease the affect of hidden nodes, RTS/CTS handshake mechanisms are employed. Due to the handshaking mechanisms some overhead and delay is introduced for each transmission.

$\mathrm{Wu}$ et al [16] presented a MDDV algorithm, based on opportunistic forwarding, geographical forwarding and trajectory based forwarding. In opportunistic forwarding, the sender selects the eligible forwarder based on the predefined knowledge of the neighbors. Trajectory based forwarding is specified extending from source to destination along which a message will be moved geographically closer to the destination (geographical forwarding).Unfortunately, this approach specifies only one forwarding trajectory that may not lead to the destination with minimum delay. To increase the system's robustness and reduce the delay, multiple diverse forwarding trajectories have to be defined.

To resolve the above mentioned problems, Zhao and cao proposed Vehicle Assisted Data Delivery (VADD) for VANETs [3]. Basically this approach deals with pull-based mechanism, which adopts relay, carry and forward strategy. When the data has to be forwarded from one place to another, then this protocol suggests that path selection should be done on the basis of high density of vehicle. Even though distance traversed through this path is more, forwarding delay will be less on this path. Forwarding is based on location (L-VADD) or direction (D-VADD). L-VADD shows better performance than all other VADD protocol when there is no routing loop occurs. When it occurs, performance affects severely and data delivery ratio decreases. Hence Multi-path VADD (M-VADD) and Hybrid VADD) H-VADD is proposed, in which Hybrid probe H-VADD is developed in which both L-VADD and D-VADD protocols are used. Firstly packet is forwarded using L-VADD protocol but as the routing loop occurs the LVADD protocol is dropped and D-VADD protocol is used.

Another interesting work carried by Zhao and Zhang Zhao, Cao [6] have proposed Data Pouring and buffering scheme for push based data dissemination. The Data Pouring (DP) scheme selects one or some road having high density and mobility of vehicles i.e. axis road (A-road) and data center delivers data not only on that road but on the crossing roads (C-roads) if the vehicles are near to the intersection on C-roads. The Data Pouring Intersection Buffering (DP-IB) mechanism uses relay and broadcast stations which are actually the buffers (IBer) [6]. These IBers are placed at the intersection points and used to store data at the intersections. In the DP-IB scheme the data has been transferred from data center to the buffers present at the intersections by this way the availability of the data is increased at the intersection and the load on the server is reduced and data delivery ratio is increased. IBers periodically rebroadcast data so that vehicles passing through C-road can receive data packets. IBers update themselves with the updated data send by data center. There may be possibility of collision between the new data item send by data center and broadcast data by IBer. To avoid this collision, broadcast period is divided into two parts.

1. Busy period in which IBer can only broadcast data

2. Idle period in which IBer only listen the forwarded data.

The broadcast cycle time at the intersection $\mathrm{T}_{\mathrm{i}}$ is used to determine Dissemination Capacity, delivery ratio of DP and DP-IB scheme. This $T_{i}$ should be less than the time taken by vehicles to go through intersection region i.e. $\mathrm{T}_{\mathrm{i}}$ to guarantee that all the vehicles moving from the intersection can receive the broadcast data. The delay in the DP scheme is more because many time receiver cannot receive data packets in a single cycle and in Reliable DP (R-DP) scheme, vehicles uses request to send/clear to send (RTS/CTS) handshakes to reduce collision and hidden node problem but due to this handshake, delay is more as it blocks the flow until it receives the acknowledge of the previous packets and in DPIB scheme the delay is more as IBer uses only idle cycle to receive the forwarded packets. The delivery ratio of DP is good for very small set of data but as size of data set increases it decreases. The R-DP and DP-IB have very high data delivery ratio for limited data set size but as the data set size increases more the delivery ratio of R-DP falls whereas DP-IB scheme keeps the same delivery ratio.

\section{CONCLUSION}

Most of the dissemination protocols do not considered the real condition of vehicular traffic, road condition and obstacles on the road. Some of the protocols need high priority for alerting the vehicles in which packet forwarding delay is not acceptable. On the other hand, several protocols can be used where slight delay is tolerable with limited bandwidth. Performance of the protocols varies 
based on data set size, traffic density, data packet size and buffer size. In this work, we have focused on push-based dissemination, where the data can be efficiently delivered from moving vehicles or fixed stations to other vehicles. And also we focused on pull-based data dissemination/access, where a vehicle is enabled to query information about specific targets. Generally speaking, the push-based approach is used to disseminate data that are useful for many people, whereas the pull-based approach is used to query data that are specific for some user. In practice, a hybrid of push/pull can be used to improve the system performance, and this will be studied in our future work.

We hope that this concise work will help to make better understanding to those researchers who are new to applications of VANETs and pave their way for developing new ideas to enhance the working of these networks.

\section{ACKNOWLEDGEMENT}

We wish to convey our sincere thanks to Professor K.V.Chalapathi Rao for motivating us to initiate the research work. And also we thank Dr.Bipin Bihari Jayasingh, for giving guidance and encouragement throughout this work.

\section{REFERENCES}

[1] Brij Bihari Dubey, Naveen Chauhan and Prashant Kumar "A Survey on Data Dissemination Techniques used in VANETs", International Journal of Computer Applications (0975 - 8887) Volume 10-No.7, November 2010.

[2] A. Gupta, V. Chaudhary, V. Kumar, B. Nishad, S. Tapaswi, "VD4: Vehicular Density-dependent Data Delivery Model in Vehicular Ad hoc Networks," in IEEE Computer Society, 2010.

[3] J.Zhao and G. Cao, "VADD: Vehicle-assisted data delivery in vehicular ad hoc networks," IEEE Transaction Vehicular Technology, Vol. 57, No. 3, pp. 1910-1922, May 2008.

[4] A. Skordylis and N. Trigoni, "Delay-bounded routing in vehicular ad-hoc networks," in Proc. ACM MOBIHOC, pp. 341-350, 2008.

[5] Y. Toor, P. Muhlethaler, A. Laouiti, and A. Fortelle, "Vehicular ad hoc networks: Applications and related technical issues," Commun. Surveys Tuts., Vol. 10, No. 3, pp. 74-88, 2008.

[6] J. Zhao, Y. Zhang, and G. Cao, "Data Pouring and Buffering on the Road: A New Data Dissemination Paradigm for Vehicular Ad Hoc Networks," in IEEE Transaction on Vehicular Technology, Vol. 56, No. 6, pp. 3266-3276, 2007.
[7] Giovanni Resta, Paolo Santi, Janos Simon, "Analysis of Multi-Hop Emergency Message Propagation in Vehicular Ad Hoc Networks", Proceedings 8th ACM international symposium on Mobile ad hoc networking and computing pp. 140149, 2007.

[8] M. Mariyasagayam, T. Osafune and M. Lenardi, "Enhanced Multi-Hop Vehicular Broadcast (MHVB) for Active Safety Applications", in Proceedings of the 7th International Conference on ITS Telecommunications (ITST 2007), pp: 1-6, Sophia-Antipolis, France, June 2007.

[9] J. Nzouonta, C. Borcea, "STEID: A Protocol for Emenency Information Dissemination in Vehicular Networks", Report, Department of Computer Science, New Jersey Institute of Technology, 2006.

[10] G. Karlsson, V. Lenders, and M. May, "Delaytolerant broadcasting," in Proceedings SIGCOMM Workshop CHANTS, Pisa, Italy, pp. 197-204, 2006..

[11] T.D.C. Little and A. Agarwal, "An Information Propagation Scheme for VANETs", in Proceedings of the $8^{\text {th }}$ IEEE Conference on Intelligent Transportation Systems (ITSC 2005), pp: 155-160, Vienna, Austria, September 2005.

[12] B. Xu, A. Ouksel, and O. Wolfson, "Opportunistic resource exchange in inter-vehicle ad hoc networks," in Proceedings IEEE International Conference MDM, pp. 4-12, 2004.

[13] G. Korkmaz, E. Ekici, F. Ozguner, and U. Ozguner, "Urban multi-hop broadcast protocol for intervehicle communication systems," in Proceedings of VANET, October 2004.

[14] Q. Xu, T. Mark, J. Ko, and R. Sengupta, "Vehicleto- Vehicle Safety Messaging in DSRC," in Proceedings of VANET, October 2004.

[15] J. Yin, T. Eibatt, G. Yeung, B. Ryu, S. Habermas, H. Krishnan, and T. Talty, "Performance Evaluation of Safety Applications over DSRC Vehicular Ad Hoc Networks," in Proceedings of VANET, October 2004.

[16] H. Wu, R. Fujimoto, R. Guensler and M. Hunter, "MDDV: A Mobility-Centric Data Dissemination Algorithm for Vehicular Networks", in Proceedings of the 1st ACM Workshop on Vehicular Ad hoc Networks (VANET 2004), pp: 47-56, Philadelphia, USA, October 2004. 